\title{
TIME DOMAIN CORRELATIONS AND GATED DETECTION OF QUANTUM SOLITONS
}

\author{
D. Levandovsky, M. Vasilyev, and P. Kumar \\ Department of Electrical and Computer Engineering \\ Northwestern University, Evanston, Illinois 60208-3118 \\ Telephone: (847) 467-2261; Fax: (847) 491-4455 \\ E-mail: dmitry@ece.nwu.edu
}

\begin{abstract}
We study the time-domain quantum-noise properties of solitons in optical fibers. Perturbation theory, including quantum description of the continuum, is used to derive a complete analytical expression for the second-order correlator of the amplitude quadrature in time domain. This correlator is subsequently used to calculate the photon-number noise reduction below the standard quantum limit by means of a time-gating filter. For propagation distances up to three soliton periods, the length at which the best noise reduction occurs, a square band-reject time filter is found to produce approximately $3.2 \mathrm{~dB}$ of squeezing.
\end{abstract}

\section{INTRODUCTION}

Recently, sub-Poissonian light was generated by frequency filtering solitons after their propagation through the fiber. ${ }^{1}$ Numerical and analytical models of these experiments were developed that are based on a positive $P$ representation, ${ }^{2}$ a back propagation approach ${ }^{3}$, or a direct method using the second-order correlator of the frequency-domain amplitude quadrature. ${ }^{4}$ An experimental study of this correlator was recently reported. ${ }^{5}$ In addition, quantum properties of 1-dimensional spatial solitons of the Kerr nonlinearity have been investigated in the context of near- and far-field spatial aperturing ${ }^{6}$ and noise amplification through four-wave mixing. ${ }^{7}$ In particular, the work in Ref. 6 shows that far-field spatial aperturing is equivalent to frequency filtering of the quantum field of temporal solitons, whereas the near-field aperturing is equivalent to modulating (time-gating) the temporal solitons before their detection by a photon-counting detector. In the latter case, to study the quantum-noise properties of the solitons, one needs to obtain the second-order correlators of the time-domain (or the near-field spatial domain) quadratures. In this paper, we derive analytical expressions for the quantum-noise correlators of the time-domain soliton quadratures from their frequency-domain counterparts, taking into account complete contribution of the continuum. ${ }^{4,8}$ We subsequently apply these correlators to the problem of quantum-noise reduction by means of time gating the soliton field. 


\section{CORRELATORS OF THE TIME-DOMAIN QUADRATURE}

We start with propagation of the modal annihilation operator $\hat{a}(\tau, \xi)$ inside an optical fiber as described by the quantum nonlinear Schrödinger equation (NLSE), which in dimensionless coordinates is

$$
\frac{\partial}{\partial \xi} \hat{a}(\tau, \xi)=i\left[\frac{1}{2} \frac{\partial^{2}}{\partial \tau^{2}}+\hat{a}^{\dagger}(\tau, \xi) \hat{a}(\tau, \xi)\right] \hat{a}(\tau, \xi) .
$$

If the operators in this equation are replaced by $c$-numbers, Eq. (1) is known to have the classical fundamental-soliton solution

$$
a(\tau, \xi)=\underline{f}_{n}(\tau) \exp (i \xi / 2) \equiv \operatorname{sech}(\tau) \exp (i \xi / 2)
$$

given here in the canonical form corresponding to the average number of photons $\langle\hat{N}\rangle=$ 2, where $\hat{N} \equiv \int \hat{a}^{\dagger}(\tau) \hat{a}(\tau) \mathrm{d} \tau$. To circumvent the problem of solving for the nonlinear evolution of the operator $\hat{a}$, we use the soliton perturbation approach developed in Ref. 8. We write the operator in the following form:

$$
\begin{aligned}
\hat{a}(\tau, \xi) & =\underline{f}_{n}(\tau) \exp (i \xi / 2)+\Delta \hat{b}(\tau, \xi) \\
& \equiv\left[\underline{f}_{n}(\tau)+\Delta \hat{a}(\tau, \xi)\right] \exp (i \xi / 2),
\end{aligned}
$$

with $\Delta \hat{a}$ subject to usual commutation relations everywhere inside the fiber

$$
\begin{gathered}
{\left[\Delta \hat{a}(\tau, \xi), \Delta \hat{a}^{\dagger}\left(\tau^{\prime}, \xi\right)\right]=\delta\left(\tau-\tau^{\prime}\right),} \\
{\left[\Delta \hat{a}(\tau, \xi), \Delta \hat{a}\left(\tau^{\prime}, \xi\right)\right]=\left[\Delta \hat{a}^{\dagger}(\tau, \xi), \Delta \hat{a}^{\dagger}\left(\tau^{\prime}, \xi\right)\right]=0 .}
\end{gathered}
$$

Substituting Eq. (3) in Eq. (1), and keeping only terms that are linear in $\Delta \hat{b}$, divides the analysis of Eq. (1) into two separate problems: the soliton solution of the classical NLSE, and the solution of the linearized operator equation

$$
\frac{\partial \Delta \hat{b}}{\partial \xi}=\frac{i}{2} \frac{\partial^{2}}{\partial \tau^{2}} \Delta \hat{b}+2 i|a(\tau, \xi)|^{2} \Delta \hat{b}+i a(\tau, \xi)^{2} \Delta \hat{b}^{\dagger}
$$

where the quantum-mechanical fluctuation operator $\Delta \hat{b}$ represents perturbation of the classical soliton field by the quantum noise. This linearization approach is valid if the photon-number noise is fairly small compared to the mean number of photons in the soliton, which is usually the case in most experiments. Note also that, since we only retain terms up to first order in $\Delta \hat{b}$, the photon-number noise is determined by the fluctuations in the amplitude quadrature (defined below) of the soliton field. Since Eq. (6) is linear, its solution can be written as a normal-mode expansion ${ }^{4,8}$

$$
\Delta \hat{a}(\tau, \xi)=\int \frac{\mathrm{d} \Omega}{2 \pi}\left[\hat{V}_{c}(\Omega, \xi) f_{c}(\Omega, \tau)+\hat{V}_{s}(\Omega, \xi) f_{s}(\Omega, \tau)\right]+\sum_{j=n, p, \tau, \theta} \hat{V}_{j}(\xi) f_{j}(\tau),
$$

wherein the four discrete modes $f_{n}, f_{p}, f_{\tau}$, and $f_{\theta}$ represent perturbations to the soliton shape due to changes in the soliton photon number, momentum (frequency), position (time), and phase, respectively; and $f_{c}$ and $f_{s}$ are the symmetric and antisymmetric parts, respectively, of the modes that represent perturbations of the continuum (dispersive radiation) in the fiber. Note that in this formalism all the $\xi$ dependence in Eq. (7) is associated with the operator coefficients $\hat{V}_{j}, \hat{V}_{c}, \hat{V}_{s}$ (Heisenberg picture), which are Hermitian. The modes $f_{j}, f_{c}, f_{s}$ used in the expansion given by Eq. (7), which are 
the same as those in Ref. 9, are properly normalized, orthogonal, and form a complete set. Thus, orthogonality of the modes and completeness of this solution allow for the reconstruction of the total quantum field at the output of the optical fiber by solving for the evolution of the operator coefficients in Eq. (7) and applying the principle of superposition. In our model we assume a white coherent-state quantum noise at the input of the fiber $(\xi=0)$, which excites all the modes of the linearized NLSE. Defining two quadrature-like operators in frequency domain as

$$
\begin{aligned}
\Delta \hat{a}_{c}(\omega, \xi) & \equiv\left[\Delta \hat{a}(\omega, \xi)+\Delta \hat{a}^{\dagger}(\omega, \xi)\right] / 2, \\
\Delta \hat{a}_{s}(\omega, \xi) & \equiv\left[\Delta \hat{a}(\omega, \xi)-\Delta \hat{a}^{\dagger}(\omega, \xi)\right] / 2,
\end{aligned}
$$

which represent amplitude (cosine) and phase (sine) perturbations of the field at a given frequency $\omega$, we may write the following expressions for the three correlators:

$$
\begin{aligned}
& G^{c c}\left(\omega, \omega^{\prime}, \xi\right) \equiv 4\left\langle\Delta \hat{a}_{c}(\omega, \xi) \Delta \hat{a}_{c}\left(\omega^{\prime}, \xi\right)\right\rangle=G_{N}^{c c}\left(\omega, \omega^{\prime}, \xi\right)+2 \pi \delta\left(\omega-\omega^{\prime}\right), \\
& G^{s s}\left(\omega, \omega^{\prime}, \xi\right) \equiv 4\left\langle\Delta \hat{a}_{s}(\omega, \xi) \Delta \hat{a}_{s}\left(\omega^{\prime}, \xi\right)\right\rangle=G_{N}^{s s}\left(\omega, \omega^{\prime}, \xi\right)-2 \pi \delta\left(\omega-\omega^{\prime}\right), \\
& G^{c s}\left(\omega, \omega^{\prime}, \xi\right) \equiv 4\left\langle\Delta \hat{a}_{c}(\omega, \xi) \Delta \hat{a}_{s}\left(\omega^{\prime}, \xi\right)\right\rangle=G_{N}^{c s}\left(\omega, \omega^{\prime}, \xi\right)-2 \pi \delta\left(\omega-\omega^{\prime}\right),
\end{aligned}
$$

wherein we have used the Fourier transform relation $\Delta \hat{a}(\omega, \xi)=\int \Delta \hat{a}(\tau, \xi) e^{i \omega \tau} \mathrm{d} \tau$, and introduced $G_{N}^{j k}\left(\omega, \omega^{\prime}, \xi\right)$ as the normal-ordered part of the respective correlator. We may also define two time-domain quadrature-like operators as

$$
\begin{aligned}
\Delta \hat{a}_{r}(\tau, \xi) & \equiv\left[\Delta \hat{a}(\tau, \xi)+\Delta \hat{a}^{\dagger}(\tau, \xi)\right] / 2, \\
\Delta \hat{a}_{i}(\tau, \xi) & \equiv\left[\Delta \hat{a}(\tau, \xi)-\Delta \hat{a}^{\dagger}(\tau, \xi)\right] / 2,
\end{aligned}
$$

which represent the amplitude (real) and phase (imaginary) perturbations of the field at time $\tau$. We note here that the inverse-Fourier transforms of the operators $\Delta \hat{a}_{c}(\omega, \xi)$ and $\Delta \hat{a}_{s}(\omega, \xi)$ are not the same as the two operators in Eqs. (13) and (14), but, instead, are given by

$$
\begin{aligned}
\Delta \hat{a}_{c}(\tau, \xi) & =\left[\Delta \hat{a}(\tau, \xi)+\Delta \hat{a}^{\dagger}(-\tau, \xi)\right] / 2, \\
\Delta \hat{a}_{s}(\tau, \xi) & =\left[\Delta \hat{a}(\tau, \xi)-\Delta \hat{a}^{\dagger}(-\tau, \xi)\right] / 2 .
\end{aligned}
$$

The three correlators in Eqs. (10)-(12) were derived analytically in Ref. 9 in terms of the normal modes of the linearized NLSE [Eq. (6)]. Their normal-ordered parts are plotted in Fig. 1(left). The three correlators of the time-domain quadratures, Eqs. (13) and (14), can be obtained from the inverse-Fourier transforms of the correlators in Eqs. (10)-(12) for the frequency-domain quadratures as follows:

$$
\begin{aligned}
G^{r r}\left(\tau, \tau^{\prime}, \xi\right) \equiv & 4\left\langle\Delta \hat{a}_{r}(\tau, \xi), \Delta \hat{a}_{r}\left(\tau^{\prime}, \xi\right)\right\rangle \equiv \delta\left(\tau-\tau^{\prime}\right)+G_{N}^{r r}\left(\tau, \tau^{\prime}, \xi\right), \\
G_{N}^{r r}\left(\tau, \tau^{\prime}, \xi\right)= & \frac{1}{2} \operatorname{Re}\left\{G_{N}^{c c}\left(\tau, \tau^{\prime}, \xi\right)+G_{N}^{c c}\left(-\tau, \tau^{\prime}, \xi\right)+G_{N}^{s s}\left(\tau, \tau^{\prime}, \xi\right)-G_{N}^{s s}\left(-\tau, \tau^{\prime}, \xi\right)\right. \\
& \left.+G_{N}^{c s}\left(\tau, \tau^{\prime}, \xi\right)+G_{N}^{c s}\left(-\tau, \tau^{\prime}, \xi\right)+G_{N}^{c s}\left(\tau^{\prime}, \tau, \xi\right)+G_{N}^{c s}\left(-\tau^{\prime}, \tau, \xi\right)\right\}, \\
G^{i i}\left(\tau, \tau^{\prime}, \xi\right) \equiv & 4\left\langle\Delta \hat{a}_{i}(\tau, \xi), \Delta \hat{a}_{i}\left(\tau^{\prime}, \xi\right)\right\rangle \equiv-\delta\left(\tau-\tau^{\prime}\right)+G_{N}^{i i}\left(\tau, \tau^{\prime}, \xi\right), \\
G_{N}^{i i}\left(\tau, \tau^{\prime}, \xi\right)= & \frac{1}{2} \operatorname{Re}\left\{G_{N}^{c c}\left(\tau, \tau^{\prime}, \xi\right)-G_{N}^{c c}\left(-\tau, \tau^{\prime}, \xi\right)+G_{N}^{s s}\left(\tau, \tau^{\prime}, \xi\right)+G_{N}^{s s}\left(-\tau, \tau^{\prime}, \xi\right)\right. \\
& \left.+G_{N}^{c s}\left(\tau, \tau^{\prime}, \xi\right)-G_{N}^{c s}\left(-\tau, \tau^{\prime}, \xi\right)+G_{N}^{c s}\left(\tau^{\prime}, \tau, \xi\right)-G_{N}^{c s}\left(-\tau^{\prime}, \tau, \xi\right)\right\},
\end{aligned}
$$



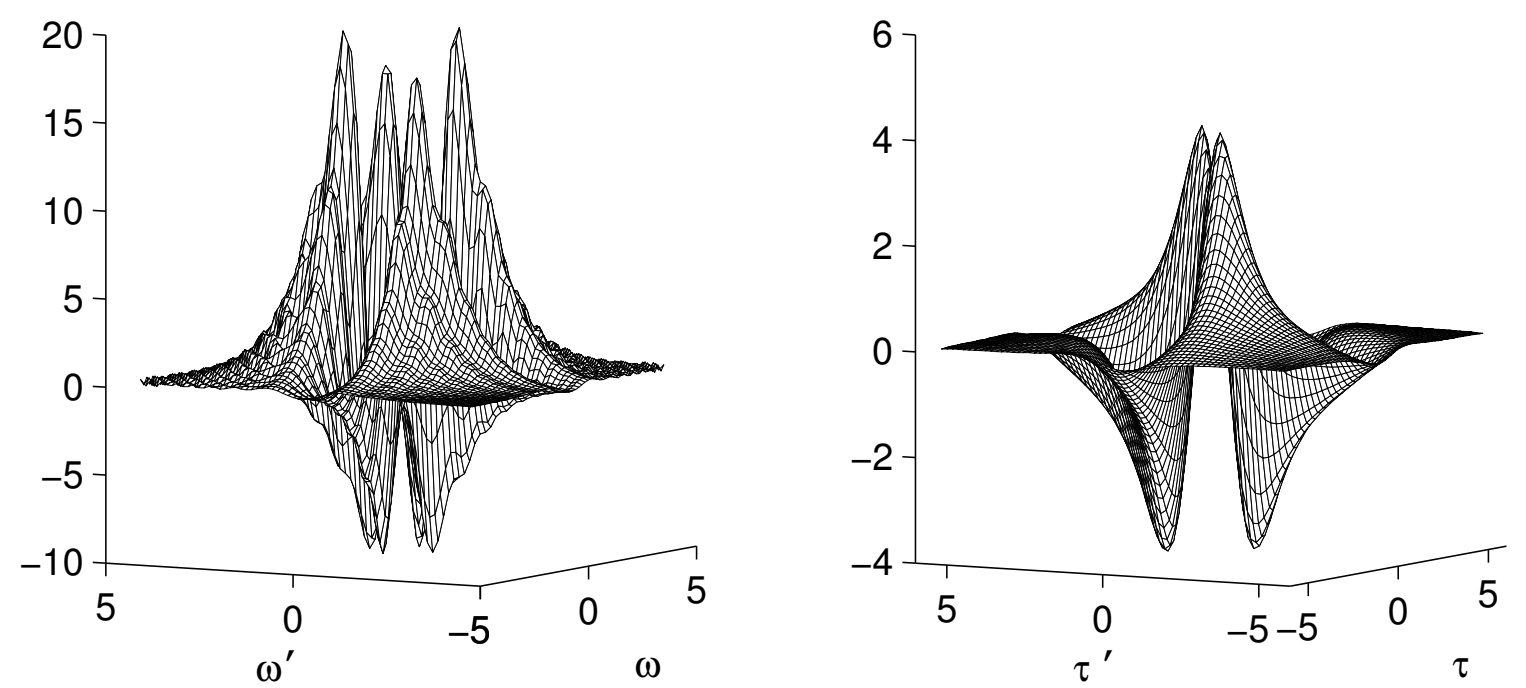

(a)
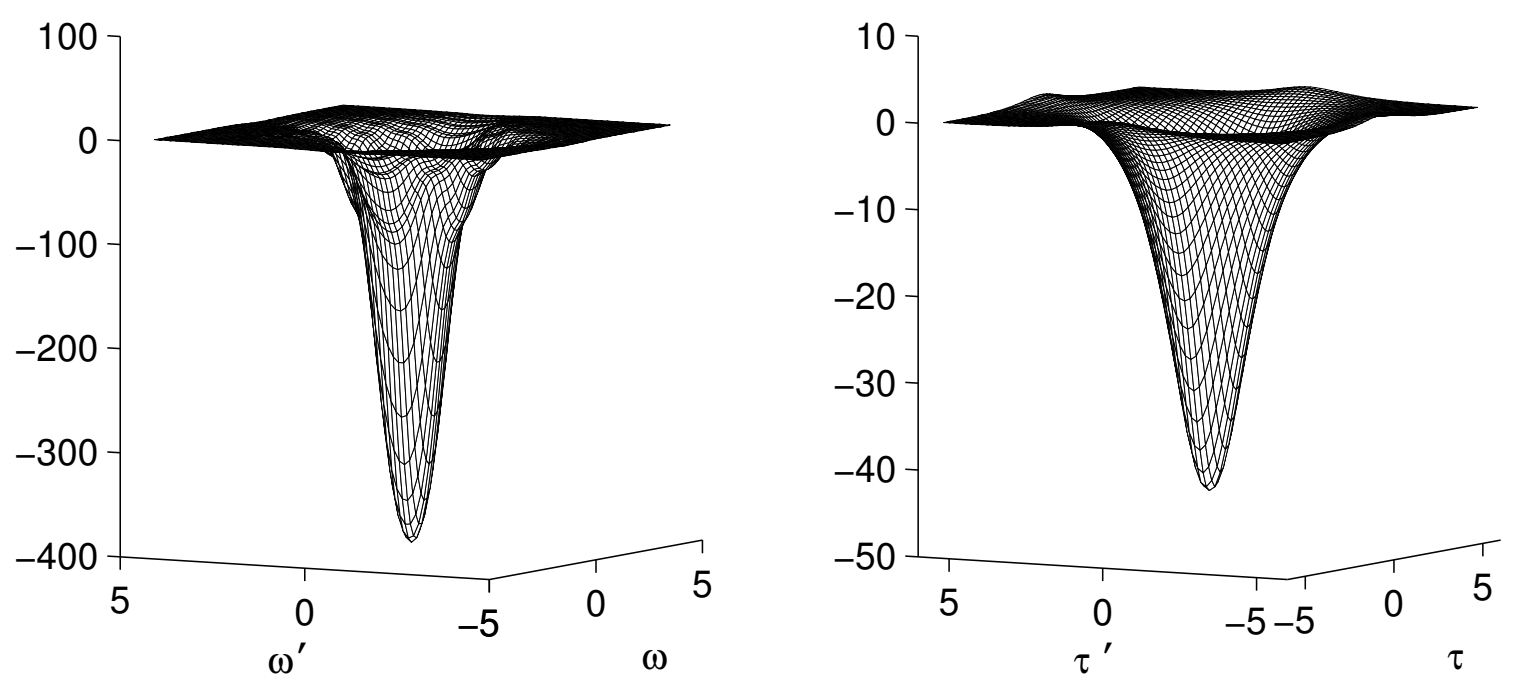

(b)
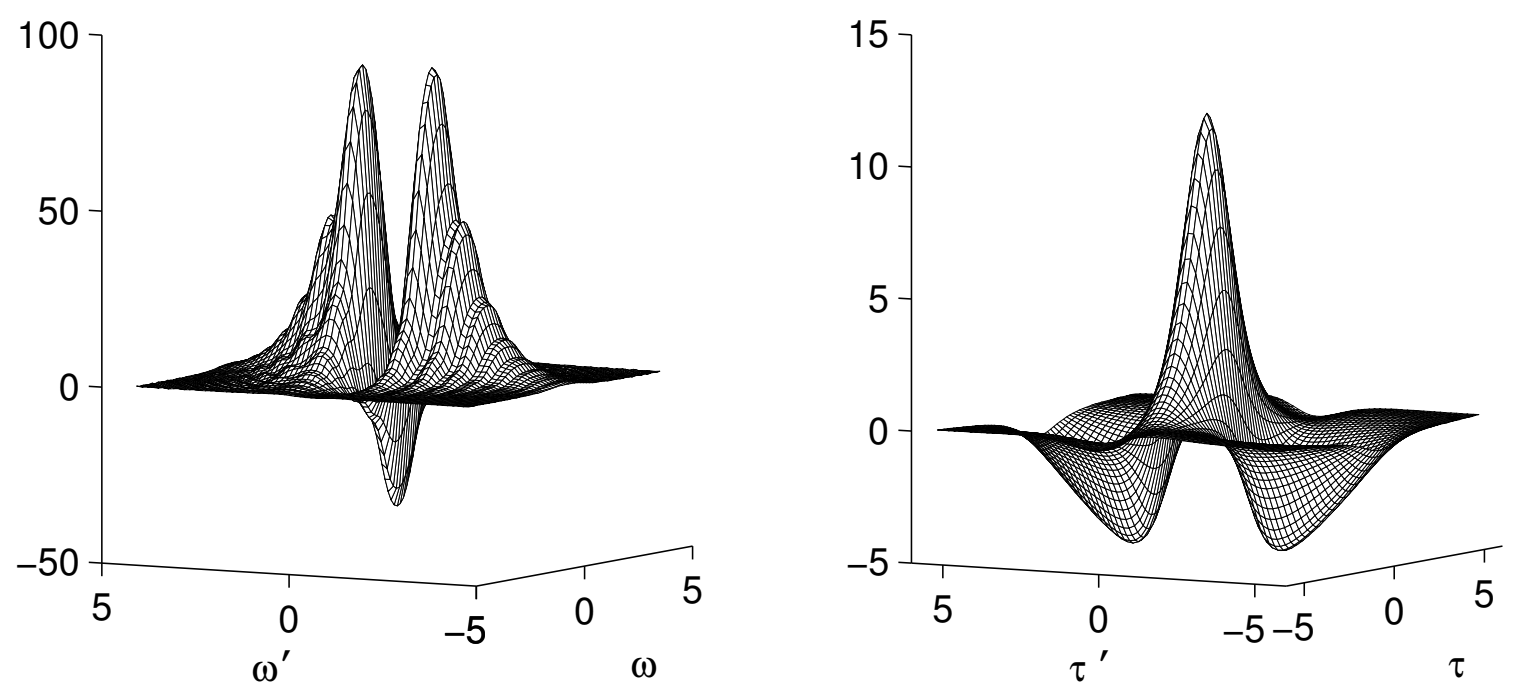

(c)

Figure 1. The three correlators of the frequency-domain (left) and time-domain (right) quadratures for a fiber length $z=3$ soliton periods: (a) $G_{N}^{c c}$ and $G_{N}^{r r}$; (b) $G_{N}^{s s}$ and $G_{N}^{i i}$; and (c) $G_{N}^{c s}$ and $G_{N}^{r i}$. 


$$
\begin{aligned}
G^{r i}\left(\tau, \tau^{\prime}, \xi\right) \equiv & 4\left\langle\Delta \hat{a}_{r}(\tau, \xi), \Delta \hat{a}_{i}\left(\tau^{\prime}, \xi\right)\right\rangle \equiv-\delta\left(\tau-\tau^{\prime}\right)+G_{N}^{r i}\left(\tau, \tau^{\prime}, \xi\right) \\
G_{N}^{r i}\left(\tau, \tau^{\prime}, \xi\right)= & \frac{i}{2} \operatorname{Im}\left\{G_{N}^{c c}\left(\tau, \tau^{\prime}, \xi\right)+G_{N}^{c c}\left(-\tau, \tau^{\prime}, \xi\right)+G_{N}^{s s}\left(\tau, \tau^{\prime}, \xi\right)-G_{N}^{s s}\left(-\tau, \tau^{\prime}, \xi\right)\right. \\
& \left.+G_{N}^{c s}\left(\tau, \tau^{\prime}, \xi\right)+G_{N}^{c s}\left(-\tau, \tau^{\prime}, \xi\right)+G_{N}^{c s}\left(\tau^{\prime}, \tau, \xi\right)-G_{N}^{c s}\left(-\tau^{\prime}, \tau, \xi\right)\right\} .
\end{aligned}
$$

The above correlators of the time-domain quadratures, which are plotted in Fig. 1(right), can now be used to solve the problem of quantum-noise reduction by means of time gating the optical field.

\section{QUANTUM-NOISE REDUCTION BY TIME GATING}

The time-gating arrangement consists of a length of optical fiber followed by an amplitude modulator. Coherent-state solitons are launched into the fiber and the emerging radiation, after passing through the modulator (time gate), is direct-detected by a photodetector. The action of a modulator, with an amplitude transfer function $H(\tau)$, on the quantum state of light is inevitably accompanied by the mixing-in of the noise of an independent vacuum-state mode $\hat{v}$, arising because of the time-dependent loss introduced. The output fluctuation operator is, then, given by

$$
\Delta \hat{a}_{\text {out }}(\tau, \xi)=|H(\tau)| \Delta \hat{a}(\tau, \xi)+\sqrt{1-|H(\tau)|^{2}} \hat{v}(\tau)
$$

wherein the amplitude transfer function must be restricted in the range $0<|H(\tau)|<1$ for it to represent a physically realizable time gate. Therefore, normalizing the output

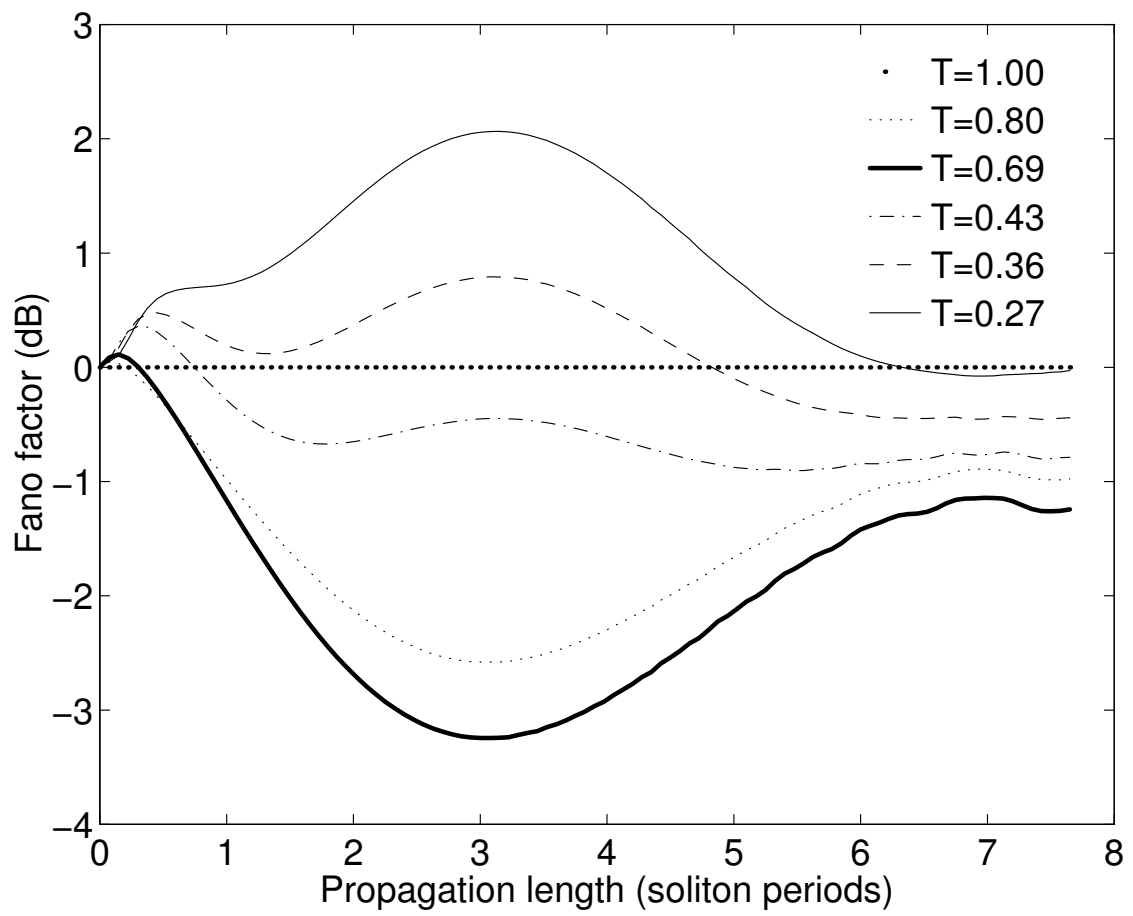

Figure 2. Fano factor for time gates of various widths $\Delta \tau$ plotted as a function of propagation distance in the fiber. The widths $\Delta \tau$ are characterized in terms of the total energy transmission $T \equiv\left\langle\hat{N}_{\text {out }}\right\rangle /\langle\hat{N}\rangle(T=1$ corresponds to the shot noise level $)$.

photon-number variance to the average output photon number, we obtain the desired Fano factor

$$
\begin{aligned}
F(\xi) & \equiv\left\langle\Delta \hat{N}_{\text {out }}(\xi)^{2}\right\rangle /\left\langle\hat{N}_{\text {out }}\right\rangle \\
& =1+\left\langle\hat{N}_{\text {out }}\right\rangle^{-1} \iint \mathrm{d} \tau \mathrm{d} \tau^{\prime} \underline{f}_{n}(\tau)|H(\tau)|^{2} G_{N}^{r r}\left(\tau, \tau^{\prime}, \xi\right)\left|H\left(\tau^{\prime}\right)\right|^{2} \underline{f}_{n}\left(\tau^{\prime}\right),
\end{aligned}
$$


where $\left\langle\hat{N}_{\text {out }}\right\rangle \equiv \int|H(\tau)|^{2} \underline{f}_{n}(\tau)^{2} \mathrm{~d} \tau$ is the average number of output photons and $G_{N}^{r r}\left(\tau, \tau^{\prime}, \xi\right)$ is the normal-ordered part of the second-order correlator of the timedomain amplitude quadrature as given by Eq. (17). This Fano factor is plotted in Fig. 2 as a function of the propagation distance $z=2 \xi / \pi$ in soliton periods for time gates of several different widths $\Delta \tau$, which reject a square region near the center of the soliton. Physical symmetry of the problem suggests that the time during which the output soliton is completely suppressed $\left[H_{\Delta \tau}(\tau)=0\right]$ must be chosen to be symmetric around the $\tau=0$ point. As seen from the figure, the best Fano factor is obtained for distances that are close to $z \approx 3$ soliton periods, where a noise reduction of $\approx 3.2 \mathrm{~dB}$ is achieved. The corresponding optimum square time gate has a total energy transmission $T \equiv\left\langle N_{\text {out }}\right\rangle /\langle N\rangle \approx 69 \%$. These results are in excellent agreement with those reported in Ref. 6.

\section{CONCLUSIONS}

In conclusion, we have derived the shapes of the three second-order correlators of the time-domain quadratures. The resulting correlations in the amplitude quadrature are found to be weaker than the spectral correlations studied in Ref. 4, although their physical origins are closely related. Namely, there exists a negative correlation between the soliton photon number and pulse width. By appropriately choosing the time gate to reject the central portion of the soliton pulse, one is able to take advantage of this correlation and reduce the photon-number noise below the standard quantum limit. This is because a greater photon number results in a narrower pulse-width, which, in turn, causes a greater photon-number loss from the time gate. In our model the correlation between the photon number and pulse width is manifested mostly through non-zero moments $\left\langle\hat{V}_{n} \hat{V}_{c}\right\rangle$ and $\left\langle\hat{V}_{n} \hat{V}_{s}\right\rangle$ at the output of the fiber, which have been given explicitly in Ref. 9.

\section{ACKNOWLEDGMENTS}

The authors acknowledge useful discussions with A. Mecozzi. This research was supported in part by the U.S. Office of Naval Research.

\section{REFERENCES}

1. S. R. Friberg, S. Machida, M. J. Werner, A. Levanon, and T. Mukai, Phys. Rev. Lett. 77, 3775 (1996); S. Spälter, M. Burk, U. Strössner, M. Böhm, A. Sizmann, and G. Leuchs, Europhys. Lett. 38, 335 (1997); S. Spälter, M. Burk, U. Strössner, A. Sizmann, and G. Leuchs, Opt. Exp. 2, 77 (1998).

2. $\quad$ M. J. Werner and S. R. Friberg, Phys. Rev. Lett. 79, 4143 (1997).

3. A. Mecozzi and P. Kumar, Opt. Lett. 22, 1232 (1997).

4. D. Levandovsky, M. Vasilyev, and P. Kumar, Opt. Lett. XX, XXX (1998).

5. S. Spälter, N. Korolkova, F. König, A. Sizmann, and G. Leuchs, Phys. Rev. Lett. 81, 786 (1998).

6. A. Mecozzi and P. Kumar, Quantum Semiclass. Opt. 10, L21 (1998)

7. E. M. Nagasako, R. W. Boyd, and G. S. Agarwal, Phys. Rev. A 55, 1412 (1997).

8. D. J. Kaup, Phys. Rev. A 42, 5689 (1990); H. A. Haus and Y. Lai, J. Opt. Soc. Am. B 7, 386 (1990); H. A. Haus, W. S. Wong, and F. I. Khatri, J. Opt. Soc. Am. B 14, 304 (1997).

9. D. Levandovsky, M. Vasilyev, and P. Kumar, Opt. Lett. XX, XXX (1998); M. Vasilyev, D. Levandovsky, and P. Kumar, submitted to Phys. Rev. A. 